\title{
Elementary Excitations in Gapped Quantum Spin Systems
}

\author{
Jutho Haegeman, ${ }^{1, *}$ Spyridon Michalakis, ${ }^{2}$ Bruno Nachtergaele,${ }^{3}$ Tobias J. Osborne, ${ }^{4}$ \\ Norbert Schuch, ${ }^{5}$ and Frank Verstraete ${ }^{1,6}$ \\ ${ }^{1}$ Department of Physics and Astronomy, Ghent University, B-9000 Ghent, Belgium \\ ${ }^{2}$ Institute for Quantum Information and Matter, Caltech, Pasadena, California 91125, USA \\ ${ }^{3}$ Department of Mathematics, University of California, Davis, California 95616, USA \\ ${ }^{4}$ Institute of Theoretical Physics, Leibniz Universität Hannover, D-30167 Hannover, Germany \\ ${ }^{5}$ Institut für Quanteninformation, RWTH Aachen, D-52056 Aachen, Germany \\ ${ }^{6}$ Vienna Center for Quantum Science, Universität Wien, A-1090 Wien, Austria
}

(Received 14 June 2013; published 19 August 2013)

\begin{abstract}
For quantum lattice systems with local interactions, the Lieb-Robinson bound serves as an alternative for the strict causality of relativistic systems and allows the proof of many interesting results, in particular, when the energy spectrum exhibits an energy gap. In this Letter, we show that for translation invariant systems, simultaneous eigenstates of energy and momentum with an eigenvalue that is separated from the rest of the spectrum in that momentum sector can be arbitrarily well approximated by building a momentum superposition of a local operator acting on the ground state. The error satisfies an exponential bound in the size of the support of the local operator, with a rate determined by the gap below and above the targeted eigenvalue. We show this explicitly for the Affleck-Kennedy-Lieb-Tasaki model and discuss generalizations and applications of our result.
\end{abstract}

DOI: 10.1103/PhysRevLett.111.080401

PACS numbers: 05.30. $-\mathrm{d}, 03.65 .-\mathrm{w}, 75.10 . J \mathrm{~m}$

Over 50 years ago, Zimmermann used the general principles of relativistic covariance and causality to show that the distinction between elementary excitations and bound states in relativistic quantum field theories is artificial and dependent on the formalism [1]. While it is tempting to call excited states "elementary" when they can be connected to the one-particle excitations of the free theory by adiabatically switching off the interaction terms, in the fully interacting theory all discrete eigenstates of the energymomentum operator $P_{\mu}$ are equivalent in that they can be created by acting on the vacuum with local operators satisfying the principle of microscopic causality.

In nonrelativistic quantum many body physics, such as quantum lattice systems, Zimmermann's result does not apply. Even for systems with translation invariance, there is no Lorentz boost to transform the energy spectrum and eigenstates in one momentum sector to a different momentum sector. Consequently, the complete energy-momentum diagram and the set of excitations are often much more complex. In addition, in many strongly correlated systems, there is no obvious free theory that can serve as a starting point to define the structure of elementary excitations. However, for systems with only local interactions in the Hamiltonian, the famous Lieb-Robinson (LR) bounds [2] can replace strict causality in, e.g., Fredenhagen's proof of the exponential clustering theorem [3], which then results in a proof for the exponential decay of correlations for the ground state of any local gapped Hamiltonian [4]. The LR bounds were also used in proving the area law for entanglement entropy of one-dimensional systems [5] and recent extensions thereof [6].
In this Letter, we extend the list of applications of the LR bounds by proving an analogue of Zimmermann's result for quantum lattice systems. For a translation invariant Hamiltonian, we show that an excitation energy that is separated by a gap from the rest of the spectrum within a given momentum sector corresponds to an eigenvector that can be approximated by the momentum superposition of a local operator acting on the ground state, with an error that is exponentially small in the size of the support of the operator. The picture to have in mind is sketched in Fig. 1. We target an energy eigenstate $\left|\Psi_{p, \alpha}\right\rangle$ with momentum $\boldsymbol{p}$ and an energy $E_{p, \alpha}$ that is separated from the other energy eigenvalues at momentum $\boldsymbol{p}$ by a gap that does not vanish in the thermodynamic limit. Because of the lack of Lorentz transformations, we cannot boost this state to any other momentum and have to treat each momentum sector
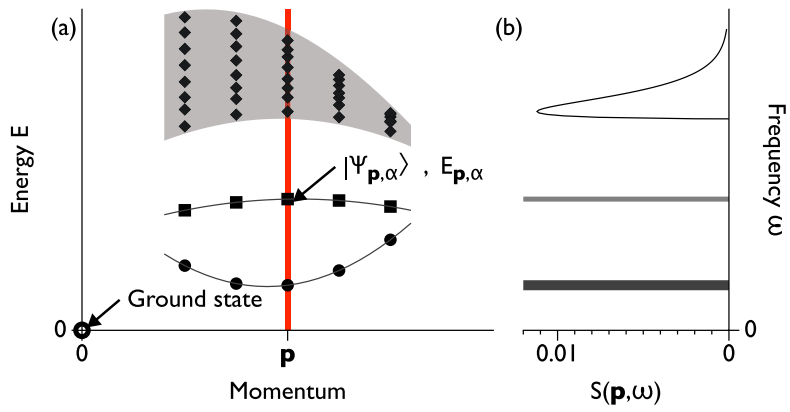

FIG. 1 (color online). Sketch of the energy-momentum diagram. The points illustrate the eigenvalues of the Hamiltonian for a finite periodic system, whereas the lines and band illustrate the distribution of eigenvalues in the thermodynamic limit. 
independently. In particular, the resulting operator that is used to build an approximation of $\left|\Psi_{p, \alpha}\right\rangle$ can itself depend on the momentum $\boldsymbol{p}$. We also discuss possible extensions and applications of our result and provide a numerical illustration for the Affleck-Kennedy-Lieb-Tasaki chain.

Let us consider a $d$-dimensional lattice $\Lambda$, which for simplicity we assume to be finite, cubic, and periodic, i.e., $\Lambda \equiv \mathbb{Z}_{N}^{d}$. Arbitrary lattice sites are denoted by $\boldsymbol{x}, \boldsymbol{y} \cdots \in$ $\Lambda$. Sets of sites are denoted by $X, Y, \ldots$; the cardinality of a set $X$ is denoted as $|X|$. Let $\|x\|$ denote the distance of a lattice point $x \in \Lambda$ to the origin $\boldsymbol{o} \in \Lambda$, with $\|\cdot\|$ a suitable distance measure on $\mathbb{Z}_{N}^{d}$. The distance between two lattice points is $\operatorname{dist}(\boldsymbol{x}, \boldsymbol{y})=\|\boldsymbol{y}-\boldsymbol{x}\|$; the distance between two sets $X, Y$ is defined as $\operatorname{dist}(X, Y)=$ $\min _{x \in X, y \in Y} \operatorname{dist}(x, y)$; and the diameter of a set $X$ is defined as $\operatorname{diam}(X)=\max _{x, y \in X} \operatorname{dist}(x, y)$. The Fourier transform of a lattice function $f: \Lambda \rightarrow \mathbb{C}$ is defined as

$$
F(\boldsymbol{p})=\frac{1}{\sqrt{|\Lambda|}} \sum_{x \in \Lambda} e^{-i p \cdot x} f(\boldsymbol{x})
$$

for any momentum vector $\boldsymbol{p} \in \mathcal{B}$, where the Brillouin zone $\mathcal{B}$ of a cubic lattice is given as $\mathcal{B}=(2 \pi / N) \Lambda$. The inverse transformation is given by

$$
f(x)=\frac{1}{\sqrt{|\Lambda|}} \sum_{p \in \mathcal{B}} F(\boldsymbol{p}) e^{i \boldsymbol{p} \cdot \boldsymbol{x}} .
$$

To all lattice sites $\boldsymbol{x} \in \Lambda$, we associate identical finitedimensional Hilbert spaces $\mathcal{H}_{x}$; the Hilbert space of the whole system is $\mathcal{H}_{\Lambda}=\boldsymbol{\bigotimes}_{x \in \Lambda} \mathcal{H}_{x}$. For all $\boldsymbol{x} \in \Lambda$, we define the space translation operator $T_{x}$. The Hamiltonian of our system is given by $H_{\Lambda}=\sum_{X \subset \Lambda} H_{X}$, where the terms $H_{X}$ are supported on $X \subset \Lambda . H_{\Lambda}$ is assumed to be translation invariant: $\forall \boldsymbol{x} \in \Lambda$ : $\left[T_{x}, H_{\Lambda}\right]=0$. This is guaranteed by $H_{X+x}=T_{x} H_{X} T_{x}^{\dagger}$. Furthermore, we assume that there exist constants $\mu, s>0$ for which

$$
\sum_{X \ni x}\left\|H_{X}\right\||X| \exp [\mu \operatorname{diam}(X)] \leq s<\infty .
$$

This allows us to use the LR bounds [2,7]

$$
\begin{aligned}
& \left\|\left[e^{i H_{\Lambda} t} A_{X} e^{-i H_{\Lambda} t}, B_{Y}\right]\right\| \\
& \quad \leq 2\left\|A_{X}\right\|\left\|B_{Y}\right\| \times|X| \exp [-\mu \operatorname{dist}(X, Y)][\exp (2 s|t|)-1]
\end{aligned}
$$

for two operators $A_{X}$ and $B_{Y}$ supported on disjoint sets $X$ and $Y$. For simplicity, we first assume that $H_{\Lambda}$ has a unique ground state $\left|\Psi_{0}\right\rangle \in \mathcal{H}_{\Lambda}$ that is necessarily translation invariant (i.e., momentum $\boldsymbol{p}=0$ ). The energy scale is chosen such that the ground state energy is zero. We discuss the case of ground state degeneracy further on. Important is the existence of a spectral gap $\Delta E$ that separates the ground state energy from the rest of the energy spectrum and does not vanish in the thermodynamic limit. All other eigenstates of $H$ are denoted as $\left|\Psi_{p, \alpha}\right\rangle$ with energy $E_{p, \alpha} \geq \Delta E$, where $p \in \mathcal{B}$ labels the momentum sector and the index $\alpha$ labels all eigenstates within a given momentum sector. They are assumed to be normalized such that $\left\langle\Psi_{p, \alpha} \mid \Psi_{p^{\prime}, \alpha^{\prime}}\right\rangle=\delta_{p, p^{\prime}} \delta_{\alpha, \alpha^{\prime}}$.

As a last preliminary, we introduce two more definitions before stating the main theorem. The Fourier transform of an operator $O$, denoted as $O(\boldsymbol{p})$, is defined by

$$
O(\boldsymbol{p})=\frac{1}{\sqrt{|\Lambda|}} \sum_{x \in \Lambda} e^{i \boldsymbol{p} \cdot \boldsymbol{x}} T_{x} O T_{x}^{\dagger} .
$$

For every bounded operator $O$ with support in the compact set $X$ and with zero ground state expectation value $\left(\left\langle\Psi_{0}|O| \Psi_{0}\right\rangle=0\right)$, we can define a momentum eigenstate $\left|\Phi_{p}[O]\right\rangle$ with momentum $\boldsymbol{p}$ by

$$
\left|\Phi_{p}[O]\right\rangle=O(p)\left|\Psi_{0}\right\rangle=\frac{1}{\sqrt{|\Lambda|}} \sum_{x \in \Lambda} e^{i p \cdot x} T_{x} O\left|\Psi_{0}\right\rangle .
$$

The exponential decay of connected correlation functions set by the spectral gap $\Delta E$ makes it straightforward to show that the norm of $\left|\Phi_{p}[O]\right\rangle$ remains finite in the thermodynamic limit. In particular, for an operator $O_{X}$ supported on $X \subset \Lambda$, the following bound follows from [4]:

$$
\|\left|\Phi_{p}\left[O_{X}\right]\right\rangle\|\leq \sqrt{\operatorname{diam}(X)+|X| / \delta}\| O_{X} \|,
$$

with $\delta \sim \Delta E$ independent of the lattice size. In the formulation of our main result below, we use the notation $B_{\ell}(X)$ for the set of sites $x$ for which $\operatorname{dist}(x, X) \leq \ell$.

Theorem 1.-Let $\left|\Psi_{p, \alpha}\right\rangle$ be a normalized momentum $p$ eigenstate of $H$ with a nondegenerate eigenvalue $E_{p, \alpha}$, such that in the thermodynamic limit $|\Lambda| \rightarrow \infty$,

$$
\left|E_{p, \alpha}-E_{p, \beta}\right| \geq \delta E \quad \forall \beta \neq \alpha .
$$

In addition, we assume there exists an operator $O$ with $\left\langle\Psi_{0}|O| \Psi_{0}\right\rangle=0$ and support in $X$, such that its Fourier transform $O(p)$ has a nonzero spectral weight $\left|\left\langle\Psi_{p, \alpha}|O(p)| \Psi_{0}\right\rangle\right| \geq f\|O\|$, with $f>0$ independent of $|\Lambda|$.

We can then define a new operator $O^{(\ell)}$ with support in $B_{\ell}(X)$, such that $\left|\Phi_{p}\left[O^{(\ell)}\right]\right\rangle$, defined in Eq. (2), satisfies

$$
F=\frac{\left|\left\langle\Psi_{p, \alpha} \mid \Phi_{p}\left[O^{(\ell)}\right]\right\rangle\right|}{\|\left|\Phi_{p}\left[O^{(\ell)}\right]\right\rangle \|} \geq 1-p_{X}(\ell) \exp \left[-\frac{\delta E}{2 v_{\mathrm{LR}}} \ell\right]
$$

for $\ell$ sufficiently large, a polynomial $p_{X}(\ell) \sim D_{X}(\ell) f^{-1}$ with $D_{X}(\ell)$ defined in Eq. (8) and $v_{\mathrm{LR}}=(\delta E / 2+2 s) / \mu$.

Hence, we can approximate the excited state by acting with the momentum superposition of a localized operator $O^{(\ell)}$ with an error that is exponentially small in the linear size of the support of the operator. Before continuing to the proof, some comments are in order. We assume that a local operator $O$ can be found for which $\left|\left\langle\Psi_{p, \alpha}|O(p)| \Psi_{0}\right\rangle\right| \geq$ $f\|O\|$ is nonzero. This factor appears as the strength of the isolated pole $\omega=E_{\alpha}$ in the (Fourier-transformed) dynamic correlation function 


$$
D(\boldsymbol{p}, \omega)=\left\langle\Psi_{0}\left|O(\boldsymbol{p})^{\dagger} \frac{1}{\omega-H_{\Lambda}+i \epsilon} O(\boldsymbol{p})\right| \Psi_{0}\right\rangle .
$$

These poles appear as $\delta$ singularities in the spectral function $S(\boldsymbol{p}, \omega) \sim \operatorname{Im} D(\boldsymbol{p}, \omega)$. The nontriviality of our result is in the fact that $f$ can be an arbitrarily small fraction and most of the spectral weight can be distributed at other isolated poles or at a continuum in the thermodynamic limit, as sketched in Fig. 1(b). The rate of exponential convergence of $F \rightarrow 1$ as a function of $\ell$ does not depend on the magnitude of $f$. The importance of connecting excitations of the system to the spectral function of local operators is that this is the main observable from which information about excitations can be obtained in experiments. Note that we are only considering isolated singularities of $S(\boldsymbol{p}, \omega)$, which correspond to eigenstates of the Hamiltonian. We are not considering quasiparticles in the sense of strong resonances in the continuum distribution of the spectral function, which are superpositions of many eigenstates with slightly different energies and thus only have a finite lifetime [8].

Proof.-We start by applying an energy filter to define $O_{1}$,

$$
O_{1}=\frac{1}{\sqrt{2 \pi q}} \int_{-\infty}^{+\infty} e^{-i H t} O e^{+i H t} e^{i E_{\alpha, p} t} e^{\left(-t^{2} / 2 q\right)} d t,
$$

so that the state $\left|\Phi_{p}\left[O_{1}\right]\right\rangle$ satisfies

$$
\left|\left\langle\Psi_{p, \alpha} \mid \Phi_{p}\left[O_{1}\right]\right\rangle\right|=\left|\left\langle\Psi_{p, \alpha} \mid \Phi_{p}[O]\right\rangle\right| \geq f\|O\|,
$$

and for any $\beta \neq \alpha$,

$$
\left|\left\langle\Psi_{p, \beta} \mid \Phi_{p}\left[O_{1}\right]\right\rangle\right| \leq e^{\left(-q \delta E^{2} / 2\right)}\left|\left\langle\Psi_{p, \beta} \mid \Phi_{p}[O]\right\rangle\right| .
$$

By restricting the time integration in the energy filtering, we obtain a new operator $\mathrm{O}_{2}$

$$
O_{2}=\frac{1}{\sqrt{2 \pi q}} \int_{-T}^{+T} e^{-i H t} O e^{+i H t} e^{i E_{\alpha, p} t} e^{\left(-t^{2} / 2 q\right)} d t .
$$

Using the triangle inequality, we obtain the bound

$$
\begin{aligned}
\left|\left\langle\Psi_{p, \alpha} \mid \Phi_{p}\left[O_{2}\right]\right\rangle\right| \geq & \left|\left\langle\Psi_{p, \alpha} \mid \Phi_{p}\left[O_{1}\right]\right\rangle\right| \\
& -\left|\left\langle\Psi_{p, \alpha} \mid \Phi_{p}\left[O_{2}-O_{1}\right]\right\rangle\right| \\
\geq & \left(1-c e^{\left(-T^{2} / 2 q\right)}\right)\left|\left\langle\Psi_{p, \alpha} \mid \Phi_{p}[O]\right\rangle\right| \\
\geq & \left(1-c e^{\left(-T^{2} / 2 q\right)}\right) f\|O\|,
\end{aligned}
$$

and for $\beta \neq \alpha$,

$$
\begin{aligned}
\left|\left\langle\Psi_{p, \beta} \mid \Phi_{p}\left[O_{2}\right]\right\rangle\right| \leq & \left|\left\langle\Psi_{p, \beta} \mid \Phi_{p}\left[O_{1}\right]\right\rangle\right| \\
& +\left|\left\langle\Psi_{p, \beta} \mid \Phi_{p}\left[O_{2}-O_{1}\right]\right\rangle\right| \\
\leq & \left(e^{\left(-q \delta E^{2} / 2\right)}+c e^{\left(-T^{2} / 2 q\right)}\right)\left|\left\langle\Psi_{p, \beta} \mid \Phi_{p}[O]\right\rangle\right|,
\end{aligned}
$$

with $c$ such that $(\pi)^{-1 / 2} \int_{|t|>T} e^{-t^{2}} d t \leq c e^{-T^{2}}$. Finally, we replace $O_{2}$ by its localized version $O^{(\ell)}$,

$$
O^{(\ell)}=\operatorname{Tr}_{\mathcal{H}_{\Lambda \backslash B_{\ell}(X)}} O_{2},
$$

by taking the normalized partial trace over the spins in $\Lambda \backslash B_{\ell}(X)$. From the LR bounds in Eq. (1), we obtain

$$
\left\|O^{(\ell)}-O_{2}\right\| \leq \frac{2 X}{s \sqrt{2 \pi q}}\|O\| \exp (2 s T-\mu \ell) .
$$

Moreover, we have $O_{2}-O^{(\ell)}=\sum_{n \geq \ell}\left(O^{(\ell+1)}-O^{(\ell)}\right)$, with $\left\|O^{(\ell+1)}-O^{(\ell)}\right\| \leq\left\|O^{(\ell+1)}-O_{2}\right\|+\left\|O_{2}-O^{(\ell)}\right\| \leq$ $(4|X| / s \sqrt{2 \pi q})\|O\| \exp (2 s T-\mu \ell)$. Together with the bound (3), from the telescoping sum we get

$$
\begin{aligned}
\|\left|\Phi_{p}\left[O^{2}-O^{(\ell)}\right]\right\rangle \| & \leq \sum_{n \geq \ell} \|\left|\Phi_{p}\left[O^{(n+1)}-O^{(n)}\right]\right\rangle \| \\
& \leq \sum_{n \geq \ell} C\left(B_{n+1}(X)\right)\left\|O^{(n+1)}-O^{(n)}\right\| \\
& \leq D_{X}(\ell)\|O\| e^{2 s T-\mu \ell},
\end{aligned}
$$

with $C(Y) \sim \sqrt{\operatorname{diam}(Y)+|Y| / \delta}, \delta \sim \Delta E$, and

$$
D_{X}(\ell) \sim|X| C\left(B_{\ell+1}(X)\right) /(s \mu \sqrt{2 \pi q}) .
$$

Using the above bound,

$$
\begin{aligned}
\left|\left\langle\Psi_{p, \alpha} \mid \Phi_{p}\left[O^{(\ell)}\right]\right\rangle\right| \geq & \left|\left\langle\Psi_{p, \alpha} \mid \Phi_{p}\left[O_{2}\right]\right\rangle\right| \\
& -\left|\left\langle\Psi_{p, \alpha} \mid \Phi_{p}\left[O^{(\ell)}-O_{2}\right]\right\rangle\right| \\
\geq & \left\{1-c e^{\left(-T^{2} / 2 q\right)}\right. \\
& \left.-\left[D_{X}(\ell) / f\right] e^{2 s T-\mu \ell}\right\} f\|O\| .
\end{aligned}
$$

Define the seminorm $\||\Phi\rangle \|^{\prime}=\left(\sum_{\beta \neq \alpha}\left|\left\langle\Psi_{p, \beta} \mid \Phi\right\rangle\right|^{2}\right)^{1 / 2}$, which satisfies $\||\Phi\rangle\left\|^{\prime} \leq\right\||\Phi\rangle \|$. Then,

$$
\begin{aligned}
\|\left|\Phi_{p}\left[O^{(\ell)}\right]\right\rangle \|^{\prime} \leq & \|\left|\Phi_{p}\left[O_{2}\right]\right\rangle\left\|^{\prime}+\right\|\left|\Phi_{p}\left[O^{(\ell)}-O_{2}\right]\right\rangle \|^{\prime} \\
\leq & \left(e^{\left(-q \delta E^{2} / 2\right)}+c e^{\left(-T^{2} / 2 q\right)}\right) \|\left|\Phi_{p}[O]\right\rangle \| \\
& +\|\left|\Phi_{p}\left[O_{2}-O^{(\ell)}\right]\right\rangle \| \\
\leq & \left(e^{\left(-q \delta E^{2} / 2\right)}+c e^{\left(-T^{2} / 2 q\right)}\right. \\
& \left.+D_{X}(\ell) e^{2 s T-\mu \ell}\right)\|O\| .
\end{aligned}
$$

Since we are trying to construct a lower bound for the fidelity $F$ from Eq. (4), we can upper bound the denominator by $\|\left|\Phi_{p}\left[O^{(\ell)}\right]\right\rangle \| \leq\left|\left\langle\Psi_{p, \alpha} \mid \Phi_{p}\left[O^{(\ell)}\right]\right\rangle\right|+$ $\|\left|\Phi_{p}\left[O^{(\ell)}\right]\right\rangle \|^{\prime}$ so that

$$
\begin{aligned}
F & \geq\left(1+\frac{\|\left|\Phi_{p}\left[O^{(\ell)}\right]\right\rangle \|^{\prime}}{\left|\left\langle\Psi_{p, \alpha} \mid \Phi_{p}\left[O^{(\ell)}\right]\right\rangle\right|}\right)^{-1} \\
& \geq 1-\frac{\|\left|\Phi_{p}\left[O^{(\ell)}\right]\right\rangle \|^{\prime}}{\left|\left\langle\Psi_{p, \alpha} \mid \Phi_{p}\left[O^{(\ell)}\right]\right\rangle\right|} .
\end{aligned}
$$

Using an upper bound for the numerator of the second term and a lower bound for its denominator, we get

$$
F \geq 1-\frac{1}{f} \frac{e^{-q \delta E^{2} / 2}+c e^{-T^{2} /(2 q)}+D_{X}(\ell) e^{2 s T-\mu \ell}}{1-c e^{-T^{2} /(2 q)}-\left[D_{X}(\ell) / f\right] e^{2 s T-\mu \ell}} .
$$


We now set $T=\ell / v_{\mathrm{LR}}, q=T / \delta E$, and $v_{\mathrm{LR}}$ such that $\mu-2 s / v_{\mathrm{LR}}=\delta E / 2 v_{\mathrm{LR}}$ in order to reproduce Eq. (4),

$F \geq 1-\left(\frac{1+c+D_{X}(\ell)}{1-\left[c+D_{X}(\ell) / f\right] e^{-\delta E \ell /\left(2 v_{\mathrm{LR}}\right)}}\right) \frac{1}{f} e^{-\delta E \ell /\left(2 v_{\mathrm{LR}}\right)}$,

with $\ell \geq \ell_{0}$, where $\left[c+D_{X}(\ell) / f\right] e^{\left(-\delta E / 2 v_{\mathrm{LR}}\right) \ell_{0}} \leq 1 / 2$.

It is easy to generalize the result above in the case where the energy eigenvalue $E_{p, \alpha}$ is (nearly) degenerate, or overlaps with other eigenvalues, but where we can isolate it by restricting to a sector with specific quantum numbers corresponding to additional symmetries of the Hamiltonian. For example, to approximate an isolated spin- $J$ multiplet of excitations of an SU(2) symmetric Hamiltonian, we can use a set of operators $O_{m}(m=-J, \ldots,+J)$ that also transforms as the spin- $J$ irreducible representation under $\mathrm{SU}(2)$.

The case of a degenerate ground state is more subtle and requires an approach in terms of operator algebras and their representations. Similar developments in the context of the Haag-Kastler framework of local quantum theory [9] do reproduce the locality of excitations [10], but it was also found that some excitations require the introduction of nonlocal fields in stringlike regions [11]. Indeed, for every ground state of the system, the Gelfand-NaimarkSegal construction provides a Hilbert space in which we can apply the same formalism, with possible modification to account for the fact that the ground state might not be translation invariant. Note that the construction above survives the thermodynamic limit, the only complication being that momentum eigenstates can no longer be normalized to 1 and should satisfy a $\delta$ normalization instead. However, there might be additional representations that do not correspond to a single ground state and which define unitarily inequivalent Hilbert spaces, known as superselection sectors, in which to look for excitations. These stringlike excitations appear as kinks in systems with symmetry breaking [12], as electric charges in gauge theories [13], or as anyonic excitations in systems with topological order $[14,15]$.

The main application of our result is that it validates constructions such as the Feynman-Bijl Ansatz [16] or the single mode approximation [17] as variational Ansätze for excitations. Given the exact ground state $\left|\Psi_{0}\right\rangle$, we can try to determine the spectrum of isolated excitations by building a linear space of states $\left|\Phi_{p}[O]\right\rangle$, where the variational parameters are encoded in the operator $O$ with support on a compact set $X$. That this even works with an approximate ground state $\left|\tilde{\Psi}_{0}\right\rangle$ was illustrated within the framework of matrix product states (MPSs) [18,19]. For the particular example of the Affleck-Kennedy-Lieb-Tasaki model [20], which has an exact MPS ground state with bond dimension $D=2$, we illustrate the exponential convergence of the excitation energy of a trial state of the form

$$
\sum_{n \in \mathbb{Z}} \sum_{s} e^{i p n} T_{n} \boldsymbol{v}_{L}^{\dagger} \cdots A^{s_{0}} B^{s_{1} s_{2}, \ldots, s_{\ell}} A^{s_{\ell+1}} \cdots \boldsymbol{v}_{R}|s\rangle,
$$

where $s=\left(s_{0}, s_{1}, \ldots\right)$ with $s_{k}=\{-1,0,+1\}$, the matrices $A^{s_{k}}$ encode the ground state, the tensor $B$ acts on a block of $\ell$ sites and contains the variational parameters, $|\boldsymbol{s}\rangle=\cdots \otimes$ $\left|s_{n}\right\rangle \otimes\left|s_{n+1}\right\rangle \otimes \cdots$ is the direct product basis, and $\boldsymbol{v}_{L, R}$ are boundary vectors which disappear in the thermodynamic limit. The variational space spanned by this Ansatz is equivalent to the set of states $\left|\Phi_{p}[O]\right\rangle$ where $O$ acts on $\ell$ sites. Figure 2(a) shows the lowest threefold degenerate excitation energy across momentum space, whereas Fig. 2(b) shows the convergence of this energy as a function of $\ell$. For the selected momenta $p>0.4 \pi$, the variational energy converges exponentially fast as a function of the block size $\ell$. Momentum $p=0.4 \pi$ is a borderline case, as it is hard to predict from the numerics whether the gap between the single-magnon dispersion curve and the multimagnon continuum is still open. Indeed, the absence of Lorentz boost symmetries allows excitations to only exist in certain subdomains of momentum space. Figure 2(c)
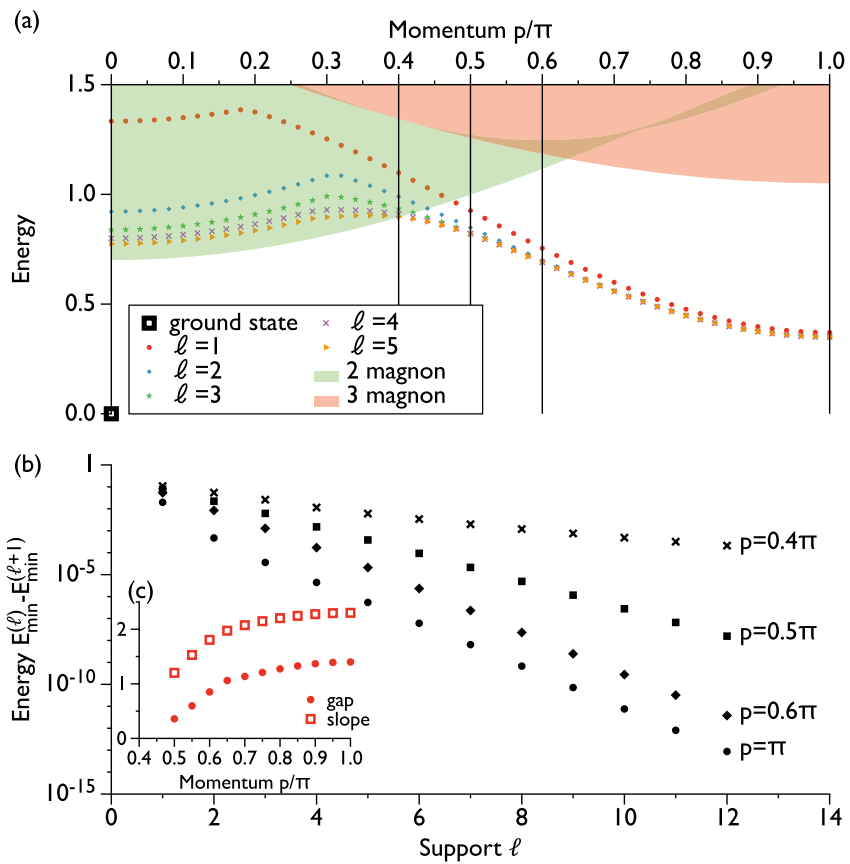

FIG. 2 (color online). Lowest variational excitation energies $E_{\min }^{(\ell)}$ obtained with the Ansatz from Eq. (10). (a) Shows the $E_{\min }^{(\ell)}$ as function of the momentum $p$ for $\ell=1, \ldots, 5$, as well as the approximate position of the two-magnon and three-magnon continuum based on the numerical results for the one-magnon dispersion relation with $\ell=5$. (b) Illustrates the exponential convergence of $E_{\min }^{(\ell)}$ by plotting $E_{\min }^{(\ell)}-E_{\min }^{(\ell+1)}$ for different values of $p$ [as indicated by vertical lines in (a)]. Inset (c) shows, as a function of $p$, the energy gap above the magnon dispersion and the two- or three-magnon continuum, as well as the exponential rate of convergence of the variational energy, corresponding to the slope of the lines in (b) (but also for additional values of the momentum). 
illustrates the qualitative proportionality between the exponential rate of convergence of the variational energy [21], which was given by $\delta E /\left(2 v_{\mathrm{LR}}\right)$ according to Eq. (4), and the energy gap $\delta E$ between the single-magnon excitation and the two- or three-magnon continuum.

If the ground state of some Hamiltonian $H_{\Lambda}$ is an exact MPS, one can use the injectivity property of MPS [18] to show that minimizing the energy with respect to a local operator $O$ acting on $\ell$ sites is equivalent to finding energy eigenstates of the same Hamiltonian on a lattice of $\ell$ sites with two added boundary sites and corresponding boundary terms. One can then show the existence of local operators $O$ with the required properties by bounding the energy of the special boundary terms [22]. Note that Theorem 1 also allows us to conclude that-in the thermodynamic limit-the entanglement entropy of isolated excitations equals that of the ground state plus $\log (2)$, corresponding to the excitation being to the left or right of the cut. The contribution of the terms spanning the cut vanishes in the thermodynamic limit, in agreement with Ref. [23].

In conclusion, we have shown that excited states of translation invariant lattice Hamiltonians for which the energy eigenvalue is isolated within a given momentum sector, and which can be detected in the spectral function of local operators, can be arbitrarily well approximated by the momentum superposition of a local operator acting on the ground state. By identifying these "elementary" excitations with single-particle states, we will show in a later publication that-in the thermodynamic limit—we can then build a Hilbert space of multiparticle excitations starting from the fully interacting vacuum and, in particular, that we can formulate the scattering problem for such excitations [24].

Discussions with Karel Van Acoleyen and Henri Verschelde are gratefully acknowledged. We acknowledge funding provided by the Institute for Quantum Information and Matter, an NSF Physics Frontiers Center with support of the Gordon and Betty Moore Foundation through Grant No. GBMF1250 and by the AFOSR Grant No. FA8750-122-0308 (S. M.), the National Science Foundation under Grant No. DMS-1009502 (B.N.), the Alexander von Humboldt Foundation (N.S.), an Odysseus Grant from the FWO Flanders (F. V. and J.H.), the FWF Grants FoQuS and Vicom (F. V.), the ERC Grants QUERG (F. V.) and QFTCMPS (T.J.O.), and by the Cluster of Excellence EXC 201 Quantum Engineering and SpaceTime Research (T. J. O.).

*Corresponding author. jutho.haegeman@ugent.be

[1] W. Zimmermann, Nuovo Cimento B 10, 597 (1958).

[2] E. Lieb and D. Robinson, Commun. Math. Phys. 28, 251 (1972); O. Bratteli and D. Robinson, Operator Algebras and Quantum Statistical Mechanics 2 (Springer, New York, 1997), 2nd ed.
[3] K. Fredenhagen, Commun. Math. Phys. 97, 461 (1985).

[4] M. B. Hastings, Phys. Rev. B 69, 104431 (2004); M. B. Hastings, Phys. Rev. Lett. 93, 140402 (2004); B. Nachtergaele and R. Sims, Commun. Math. Phys. 265, 119 (2006); M. Hastings and T. Koma, Commun. Math. Phys. 265, 781 (2006).

[5] M. Hastings, J. Stat. Mech. (2007) P08024.

[6] E. Hamza, S. Michalakis, B. Nachtergaele, and R. Sims, J. Math. Phys. (N.Y.) 50, 095213 (2009); S. Michalakis, arXiv:1206.6900.

[7] B. Nachtergaele, Y. Ogata, and R. Sims, J. Stat. Phys. 124, 1 (2006); M. B. Hastings, arXiv:1008.5137.

[8] L. D. Landau, Zh. Eksp. Teor. Fiz. 30, 1058 (1956) [Sov. Phys. JETP3, 920 (1957)]; L. D. Landau, Zh. Eksp. Teor. Fiz. 32, 59 (1957) [Sov. Phys. JETP5, 101 (1957)]; R. D. Mattuck, A Guide to Feynman Diagrams in the Many-Body Problem (Dover, New York, 1992), 2nd ed.

[9] R. Haag and D. Kastler, J. Math. Phys. (N.Y.) 5, 848 (1964).

[10] S. Doplicher, R. Haag, and J. E. Roberts, Commun. Math. Phys. 23, 199 (1971); 35, 49 (1974).

[11] D. Buchholz and K. Fredenhagen, Commun. Math. Phys. 84, 1 (1982).

[12] D. Schlingemann, Rev. Math. Phys. 08, 301 (1996); 08, 1187 (1996); 10, 851 (1998).

[13] K. Fredenhagen and M. Marcu, Commun. Math. Phys. 92, 81 (1983); J.A.C.A. Barata and K. Fredenhagen, Commun. Math. Phys. 113, 403 (1987); J. A.C. A. Barata and F. Nill, Commun. Math. Phys. 171, 27 (1995); 191, 409 (1998).

[14] J. Fröhlich and P. A. Marchetti, Lett. Math. Phys. 16, 347 (1988); J. Fröhlich and F. Gabbiani, Rev. Math. Phys. 02, 251 (1990); K. Szlachányi and P. Vecsernyés, Commun. Math. Phys. 156, 127 (1993); F. Nill and K. Szlachányi, Commun. Math. Phys. 187, 159 (1997).

[15] P. Naaijkens, Commun. Math. Phys. 303, 385 (2011); Rev. Math. Phys. 23, 347 (2011); arXiv:1303.4420.

[16] A. Bijl, J. de Boer, and A. Michels, Physica (Amsterdam) 8, 655 (1941); R. P. Feynman, Phys. Rev. 94, 262 (1954); R. P. Feynman and M. Cohen, Phys. Rev. 102, 1189 (1956).

[17] S. M. Girvin, A. H. MacDonald, and P. M. Platzman, Phys. Rev. B 33, 2481 (1986); D. P. Arovas, A. Auerbach, and F. D. M. Haldane, Phys. Rev. Lett. 60, 531 (1988).

[18] M. Fannes, B. Nachtergaele, and R. F. Werner, Commun. Math. Phys. 144, 443 (1992); F. Verstraete, J. I. Cirac, and V. Murg, Adv. Phys. 57, 143 (2008); J. I. Cirac and F. Verstraete, J. Phys. A 42, 504004 (2009); U. Schollwöck, Ann. Phys. (Amsterdam) 326, 96 (2011).

[19] E. Bartel, A. Schadschneider, and J. Zittartz, Eur. Phys. J. B 31, 209 (2003); S. G. Chung and L. Wang, Phys. Lett. A 373, 2277 (2009); B. Pirvu, J. Haegeman, and F. Verstraete, Phys. Rev. B 85, 035130 (2012); J. Haegeman, B. Pirvu, D. J. Weir, J. I. Cirac, T. J. Osborne, H. Verschelde, and F. Verstraete, Phys. Rev. B 85, 100408 (2012); J. Haegeman, T. J. Osborne, and F. Verstraete, arXiv:1305.1894.

[20] I. Affleck, T. Kennedy, E. H. Lieb, and H. Tasaki, Phys. Rev. Lett. 59, 799 (1987); Commun. Math. Phys. 115, 477 (1988).

[21] The variational error on the excitation energy can be shown to be proportional to $1-F$.

[22] S. Michalakis et al. (unpublished).

[23] I. Pižorn, arXiv:1202.3336.

[24] J. Haegeman et al. (unpublished). 